\title{
Correlation analysis between CT findings and duration of initial symptoms in young and middle- aged patients with novel coronavirus pneumonia
}

Liaoyi Lin

Department of Radiology, The First Affiliated Hospital of Wenzhou Medical University, Wenzhou, Zhejiang, 325000, China

\section{Shuangli Chen}

Department of Radiology, The First Affiliated Hospital of Wenzhou Medical University, Wenzhou, Zhejiang, 325000, China

\section{Chongyong Xu}

Department of Radiology, The Second Affiliated Hospital of Wenzhou Medical University, Wenzhou, Zhejiang, 325000, China

\section{Guoquan Cao}

Department of Radiology, The Second Affiliated Hospital of Wenzhou Medical University, Wenzhou, Zhejiang, 325000, China

\section{Jiejie Tao}

Department of Radiology, The First Affiliated Hospital of Wenzhou Medical University, Wenzhou, Zhejiang, 325000, China

\section{Houzhang Sun}

Department of Radiology, The First Affiliated Hospital of Wenzhou Medical University, Wenzhou, Zhejiang, 325000, China

\section{Gangze Fu}

Department of Radiology, The First Affiliated Hospital of Wenzhou Medical University, Wenzhou, Zhejiang, 325000, China

\section{Dingping Huang}

Department of Radiology, The First Affiliated Hospital of Wenzhou Medical University, Wenzhou, Zhejiang, 325000, China

\section{Andan Qian}

Department of Radiology, The First Affiliated Hospital of Wenzhou Medical University, Wenzhou, Zhejiang, 325000, China

\section{Peipei Pang}

GE Healthcare, Hangzhou, Zhejiang, 310000, China

\section{Xiaoming Yang}

Department of Radiology, Lab-Yang (X.M.Y.), University of Washington, Seattle, WA, USA 


\section{Jinglin Xia}

Department of Oncology, The First Affiliated Hospital of Wenzhou Medical University, Wenzhou, Zhejiang, 325000, China

\section{MeiHao Wang ( $\nabla$ wzwmh@wmu.edu.cn)}

Department of Radiology, The First Affiliated Hospital of Wenzhou Medical University, Wenzhou, Zhejiang, 325000, China

\section{Yunjun Yang ( $\nabla$ yyjunjim@163.com )}

Department of Radiology, The First Affiliated Hospital of Wenzhou Medical University, Wenzhou, Zhejiang, 325000, China

\section{Research Article}

Keywords: Tomography, X-ray computed, Signs and Symptoms, Coronavirus, Pneumonia

Posted Date: March 3rd, 2020

DOI: https://doi.org/10.21203/rs.3.rs-15760/v1

License: (c) (i) This work is licensed under a Creative Commons Attribution 4.0 International License. Read Full License 


\section{Abstract}

Objective: To retrospectively analyze the correlation between CT findings and duration of initial symptoms in young and middle-aged patients with novel coronavirus pneumonia.

Materials and methods: From January 17, 2020 to February 4, 2020, of the 54 SARS-COV-2 infection cases, 48 cases $(48 / 54,88.8 \%)$ were young and middle-aged patients under 60 years old. Finally, thirtythree patients under 60 years old with CT imaging were enrolled in this study, including 20 men and 13 women. Patients' clinical data, including gender, age, infection exposure history, heating temperature, the initial symptoms and the duration, were recorded. The CT imaging features of pneumonia was scored. The number of lung lobe involvement and the length of the largest lesion were recorded. All above CT findings and clinical data were evaluated.

Results: The median duration of initial symptoms was 3 days (range 0-7 days). The CT score and the length of the largest lesion showed statistically significant between the groups of initial symptom duration $<3$ days and initial symptom duration $\geq 3$ days $(P<0.05)$. while age, gender, infection exposure history, fever temperature and the number of affected pulmonary lobes between the two groups had no significant difference $(P>0.05)$. The duration of the initial symptom was positively correlated with both the CT score of pneumonia $(r=0.502, P=0.003)$ and the length of the largest pneumonia lesion in the lung $(r=0.506, P=0.003)$.

Conclusion: The severity of pneumonia and the size of lesions were positively correlated with the duration of the initial symptom in young and middle-aged patients.

\section{Introduction}

In December 2019, a group of patients with unknown cause pneumonia were found to be linked to a seafood wholesale market in Wuhan, China. The emergence of the infectious pneumonia caused great concern. Through whole-genome sequencing of pneumonia patients, a previously unknown novel coronavirus was discovered, which was soon identified by the world health organization (WHO) as the virus responsible for the outbreak and tentatively named 2019-nCOV $(1,2)$. phylogenetic analysis suggests that bats might be the original host of this virus. Importantly, structural analysis suggests that 2019-nCoV might be able to bind to the angiotensin-converting enzyme 2 receptor in humans (3). The novel coronavirus was then officially named "Severe Acute Respiratory Syndrome Coronavirus 2" (SARSCOV-2), and the disease caused by the coronavirus was identified as "Corona Virus Disease 2019" (COVID-19).

Some studies showed that the common symptoms at onset included fever, cough and myalgia or fatigue, and the less common symptoms included phlegm, headache, hemoptysis and diarrhea. In addition, the chest imaging examination demonstrated inflammatory changes, and some patients have underlying diseases, including diabetes, hypertension and cardiovascular diseases (4). To the best of our knowledge, study of pulmonary inflammatory lesions imaging findings in the novel coronavirus pneumonia patients 
is not much. Disease progression is associated with patient physical condition, and the elderly patients or the patients with underlying disease may increase susceptibility to novel coronavirus, speed up the progress of the disease, while young and middle-aged patients under 60 years old have few basic diseases and few pulmonary lesions. In this study, we aimed to analyze the correlation between CT findings and the duration of initial symptoms in young and middle-aged patients with novel coronavirus pneumonia. Our study may reveal the correlation of the pneumonia CT manifestations and course of the disease, and provide valuable information for pulmonary lesion changes of young and middle-aged COVID-19 patients with different duration of initial symptoms.

\section{Materials And Methods}

\section{Patients}

This retrospective study was approved by the ethics committee, and the written informed consent was waived.

54 patients were confirmed with the positive for SARS-COV-2 nucleic acid kit in the first and second affiliated hospital of Wenzhou medical university from Jan. 17, 2020 to Feb. 4, 2020. Inclusion criteria included: (1) patients under 60 years old (5); (2) Chest CT examination was conducted in our hospital and the CT imaging data was complete. (3) the basic clinical data of the patient was complete, and the medical history was clear. Among the 54 patients, 5 patients underwent $\mathrm{CR}$ examination without $\mathrm{CT}$ examination, 13 patients did not undergo CR or CT examination (including 1 patient aged 81 and 2 patients aged 66), 3 patients aged over 60 had CT examination, and 33 patients aged below 60 had CT examination and CT image data was complete. Forty-eight cases $(48 / 54,88.8 \%)$ were young and middleaged patients under 60 years old. According to the inclusion criteria, 33 patients were finally included in the study (Figure 1), including 20 males and 13 females. All patients' clinical data, including gender, age, history of infection exposure, fever temperature, initial symptoms and the duration, were recorded.

\section{CT examination and image evaluation}

The CT scans was performed in the 33 patients without administration of contrast material. 17 patients from the first affiliated hospital of Wenzhou medical university and imaged on LightSpeed VCT (GE Medical Systems, Milwaukee, WI) with the following parameters: $1.25 \mathrm{~mm}$ section thickness, $120 \mathrm{kV}$, and $350 \mathrm{~mA}$. 16 patients from the second affiliated hospital of Wenzhou medical university and imaged on Brilliance 16 (Philips Healthcare, DA Best, the Netherlands) with the following parameters: $5 \mathrm{~mm}$ section thickness, $120 \mathrm{kV}$, and $150 \mathrm{mAs}$. All examinations were performed in the supine position and with breathholding following inspiration. The scanning ranged from the tip of the lung to the level of the costophrenic angle. After scans, all the image data was transmitted to PACS system for observation and analysis. 
All CT images were reviewed by two fellowship-trained cardiothoracic radiologists with 5 years of experience with a final finding reached by consensus when there was a discrepancy. The clinical information or clinical progress of the patients were blinded to them. A semi-quantitative analysis was used to evaluate the morphology, distribution and the range of lung lesions in order to objectively reflect the lung tissue damage. CT findings of inflammation included ground glass opacity, consolidation, or both co-exist, and may be accompanied by interlobular septal thickening. Ground glass opacities were defined as hazy areas of increased opacity or attenuation without concealing the underlying vessels. Consolidation was defined as homogeneous opacification of the parenchyma obscuring the underlying vessels (6). The CT findings of pneumonia were graded on a 3-point scale: 0 as normal attenuation, 1 as ground-glass attenuation, and 2 as consolidation (7). The affected lung parenchyma was assigned to the following score according to lobes (each patient had a total of five lobes) and the extent of involvement: 0 as none, 1 as $<5 \%, 2$ as $5-25 \%, 3$ as $25-50 \%, 4$ as $50-75 \%$, and 5 as $>75 \%$. The CT scan was reviewed on the cross-sectional image. The five-point scale of the lung parenchyma distribution was then multiplied by the CT findings of pneumonia scale described above. Scores from all regions were added to obtain the final total cumulative score, with CT scores ranging from 0 to 50 (8). The number of affected pulmonary lobes and the length of the largest lesion were also recorded.

\section{Statistical analysis}

SPSS 24.0 software was used for statistical analysis. The continuous variables were expressed as mean \pm standard deviation (SD), or median and range, and the categoric variables as a number of individuals with percentage in each group. The comparisons of continuous variables between groups were performed using the Mann-Whitney $\mathrm{U}$ test. Frequencies of categoric variables were compared using the Fisher exact test. The spearman correlation analysis was used to investigate the correlation between the CT score of pulmonary inflammatory lesions and the duration of initial symptoms, as well as the correlation between the length of the largest inflammatory lesions in the lungs. $p$ values $<0.05$ were considered statistically significant.

\section{Results}

\section{Patient data}

Among the 54 confirmed SARS-COV- 2 infection cases, 48 cases $(48 / 54,88.8 \%)$ were young and middleaged patients under the age of 60 , and 33 cases were included according to the inclusion criteria. Of these 33 patients, including 20 men and 13 women, their median age was 46 years old (ranged from 28 to 57 years old). Twenty patients had exposure to infection, and 13 patients did not have exposure history. Their average fever temperature was $37.87 \pm 0.60^{\circ} \mathrm{C}$. The initial symptoms include 2 cases $(6.1 \%)$ with abdominal pain or diarrhea, 1 case $(3.0 \%)$ with sore throat, 3 cases $(9.1 \%)$ with muscle soreness or 
lack of power, 6 cases (18.2\%) with cough or cough with fever, 20 cases $(60.6 \%)$ with fever, and one patient (3.0\%区without symptoms. The median duration of initial symptoms was 3 days (range 0-7 days).

Lung lesions in CT characterized by multiple in the lung, the regional or scattered inflammatory opacity, CT finding showed the opacity as small patch ground-glass attenuation and may with interstitial changes, or consolidation of the lung, the pathological changes in the lung periphery. From the CT images, we observed that there were 3 patients $(9.1 \%)$ with normal CT manifestations, 19 patients (57.6\%) with dominated ground glass opacity, and 11 patients (33\%) with consolidation or dominated consolidation surrounded by ground glass opacity (Figure 2). The mean CT score was $5.61 \pm 4.83$ points. The mean length of the maximum inflammatory lesion in the lung was $26.00 \pm 17.62 \mathrm{~mm}$. The median number of involved lobar was 3. None lobe was involved in 4 patients $(12.1 \%)$, one lobe was involved in 6 patients (18.2\%), two lobe was involved in 5 patients (15.2\%), three lobe was involved in 5 patients $(15.2 \%)$, four lobe was involved in 7 patients $(21.2 \%)$, and five lobe was involved in 6 patients $(18.2 \%)$. The right upper lobe was involved in 13 patients (39.4\%), the right middle lobe was involved in 11 patients (33.3\%), the right lower lobe was involved in 23 patients (69.7\%), the left upper lobe was involved in 20 patients $(60.6 \%)$, and the left lower lobe was involved in 22 patients $(66.7 \%)$. Both lower lobes were involved in 20 patients $(60.6 \%)$.

According to the median duration of initial symptoms, patients were divided into two groups: initial symptom duration $<3$ days and initial symptom duration $\geq 3$ days. The results showed that $\mathrm{CT}$ scores and length of the maximum inflammatory lesion between the two groups were statistically different $(\mathrm{P}<$ 0.05), while age, gender, infection exposure history, fever temperature and the number of affected pulmonary lobes between the two groups had no significant difference (table 1).

\section{Correlation analysis}

Spearman correlation analysis showed that there was a positive correlation between the duration of the initial symptom and the CT score of lung lesions; the longer the duration of the initial symptom was, the more serious the CT manifestations of lung lesions was. The duration of the initial symptom was also positively correlated with the length of the largest inflammatory lesion in the lung; the longer the duration of the initial symptom was, the larger the lesion was (table 2).

\section{Discussion}

A novel coronavirus (SARS-COV-2) pneumonia caused multiple infections in the central Chinese city, Wuhan, and it has been shown to have human-to-human transmission and sustained human-to-human transmission characteristic, which is a new risk to public health. Although the time between the onset of the disease and seeking medical attention is now generally shorter, with $27 \%$ of patients seeking medical attention within two days of onset, but at least $89 \%$ of patients are not hospitalized until the fifth day of illness (9). Because pneumonia is the common characteristic of SARS-COV-2 infection, and it is a concern 
that the course of disease development of pneumonia in COVID-19 patients. Applying CT examination to the COVID-19 patients or suspected patients is an effective way to evaluate patient's condition. And some cases with positive chest CT findings may present with negative results of real time reversetranscription-polymerase chain- reaction (RT-PCR) for SARS-COV-2, but these patients were eventually confirmed with SARS-COV-2 infection by repeated swab tests $(10,11)$. Chest CT scan is an important inspection method. Early the pulmonary inflammatory lesions present as multiple small patchy and interstitial change, lesions in the lung periphery is obvious. Then inflammatory lesions develop into multiple infiltrates ground glass opacity, seriously even appear consolidation in the lung (12). Elderly people over 60 years old may be accompanied by other basic diseases, which will make pneumonia progress faster, while young people under 60 years old have fewer basic diseases, and pneumonia changes are less affected by other diseases. Our study showed that the CT scores and length of maximum pulmonary inflammatory lesions in the young and middle-aged patients under 60 years old with COVID-19 was statistically different between the different duration time of initial symptoms groups. The duration of the initial symptom was found to be positively correlated with the CT score of pulmonary lesions and the length of the largest inflammatory lesions in the lungs. The longer the duration of the initial symptom was, the higher the CT score was, the more serious the CT manifestations of pulmonary lesions were, the larger the lesions were.

Viruses are common causes of respiratory infections. The imaging manifestations of viral pneumonia are diverse and overlap with other infectious and inflammatory pulmonary diseases (13). Viruses in the same virus family have similar pathogenesis. Since the outbreak of Severe Acute Respiratory Syndrome (SARS) in 2002 and Middle East Respiratory Syndrome (MERS) in 2012, the possibility of coronavirus transmission from animals to humans has been confirmed (14). SARS-COV-2 is similar to the pathological process of SARS and MERS. Peripheral lung involvement was observed in SARS and MERS patients, ground glass opacity and consolidation were the main CT manifestations. The CT findings of SARS usually included ground glass opacity and consolidation in areas of unilateral or bilateral lung (15). The role of thin-slice CT in recording SARS parenchymal anomalies has also been demonstrated when chest radiographs show normal or only suspicious anomalies $(16,17)$. In this study, CT examination of COVID-19 patients showed that the lesions were mostly located in the peripheral pulmonary zone and were presented as localized or multiple patchy ground glass opacity or solid lesions. Since the viral infection was mainly interstitial lesions, there was no trend of pulmonary segments and subsegment distribution. Other studies (12) suggested that there was no significant difference in lobar or craniocaudal distribution of the novel coronavirus pneumonia.

CT findings of pulmonary inflammatory are related to the development of inflammatory pathology. In the early stage of the disease, localized inflammatory infiltration of both sides of the lungs was commonly observed, which was the most of ground glass opacity under the pleura. The early stage lung tissues showed different degree of alveolar injury, including alveolar congestion, inflammatory exudation and formation of hyaline membrane (18). Therefore, the CT manifestations showed thin ground glass opacity, and crazy-paving pattern or reticulation changes were formed when the lesions accompanied by pulmonary interstitial edema. The main CT manifestations in the progressive stage were increased 
number of lesions and expanded range, gradually involving multiple lobes in lung, and consolidation or co-existence of consolidation and ground glass opacity. Irregular consolidation is often associated with a large number of exfoliated epithelium and foam cells in the alveoli, inflammatory cell infiltration, inflammatory exudation filling the alveolar cavity. Consolidation tissues may be accompanied by capillary congestion and necrosis of lung tissue and alveoli (19). After the onset of initial symptom, the pulmonary lesions gradually developed from normal or blurred ground glass opacity to solid opacity. The CT score showed that the overall pulmonary inflammatory lesions increased after the duration of initial symptom increased, and CT scores were higher in the group of initial symptom duration $\geq 3$ days. The longer the duration of the initial symptom was, the more pneumonia infiltration and expansion will occur, and the more consolidation changes will occur.

The main symptom of SARS patients included fever, general discomfort, muscle pain, fatigue and head ache, and some patients in the acute phase might had viremia (20). The main symptom of COVID-19 patients was fever. In this study $60.6 \%$ of the patients had initial symptoms of fever. Other initial symptoms included diarrhea or a sore throat, etc. The correlation analysis showed that the duration of initial symptoms of patients was positively correlated with the CT score of pulmonary inflammatory lesions. The initial symptom duration of pneumonia may be used to evaluate disease progress, and it was associated with the size of the largest lesions. Therefore, patients with fever or other symptoms should be paid attention to. In this study, it was suggested that most of the lung lesions were blurred ground glass opacity dominated within 7 days after the initial symptoms, accounting for $57.6 \%$. The inflammatory lesions were relatively mild, while main consolidation or multiple consolidation accompanied by ground glass opacity accounted for $1 / 3$. Gaik et al. (21) showed that within the 1 st week after onset of symptoms, the main abnormalities included ground glass opacities (ten [56\%] of 18) and consolidation (eight [44\%] of 18). Ground glass opacities alone or with superimposed interlobular septal thickening were most commonly found in the 1 st week after onset of symptoms. Previous studies demonstrated that consolidation lesions on CT of viral pneumonia had a more severe clinical course than those with ground glass opacity, which might be related to further diffuse alveolar damage in the solid lesions (22). The longer the initial symptoms, the more pulmonary lesions will progress, so early treatment is of great importance for patients to control the disease.

There were several limitations in our study. First, this was a retrospective study and the number of patients enrolled was small. Only 33 young and middle-aged COVID-19 patients with CT imaging. We limited our study to chest CT, because CT was more sensitive to early or mild disease. However, in medical front lines, chest radiography may also serve as a screening tool in areas with high disease prevalence and limited resources. Second, the study was only conducted during early and mild stage, and study in the acute phase of SARS-COV-2 was missed. It is necessary to further study the imaging manifestations of severe or treated patients in the future to enrich the imaging studies of SARS-COV-2.

In conclusion, we revealed a potential positive correlation between the duration of the initial symptoms and the degree and size of pneumonia lesions. Most of the early lesions were ground glass opacity. 


\section{Acknowledgement}

no fund to support this research.

\section{Reference}

1. Zhu N, Zhang D, Wang W, et al. A Novel Coronavirus from Patients with Pneumonia in China, 2019. The New England journal of medicine. 2020. https://doi.org/10.1056/NEJMoa2001017. Published online Jan 24, 2020.

2. Gralinski LE, Menachery VD. Return of the Coronavirus: 2019-nCoV. Viruses. 2020;12(2) :E135..

3. Lu R, Zhao X, Li J, et al. Genomic characterisation and epidemiology of 2019 novel coronavirus: implications for virus origins and receptor binding. Lancet. 2020. https://doi.org/ 10.1016/S01406736(20)30251-8. Published online Jan 30, 2020.

4. Huang C, Wang Y, Li X, et al. Clinical features of patients infected with 2019 novel coronavirus in Wuhan, China. Lancet. 2020. https://doi.org/10.1016/S0140-6736(20)30183-5. Published online Jan $24,2020$.

5. Carlé A, Pedersen IB, Knudsen N, et al. Hypothyroid Symptoms Fail to Predict Thyroid Insufficiency in Old People: A Population-Based Case-Control Study. Am J Med. 2016;129(10):1082-92.

6. Hansell DM, Bankier AA, MacMahon H, McLoud TC, Müller NL, Remy J. Fleischner Society: glossary of terms for thoracic imaging. Radiology. 2008;246(3):697-722.

7. Feng $F$, Jiang $Y$, Yuan $M$, et al. Association of radiologic findings with mortality in patients with avian influenza H7N9 pneumonia. PLoS One. 2014;9(4):e93885.

8. Chang Y-C, Yu C-J, Chang S-C, et al. Pulmonary sequelae in convalescent patients after severe acute respiratory syndrome: evaluation with thin-section CT. Radiology. 2005;236(3):1067-75. 
9. Li Q, Guan X, Wu P, et al. Early Transmission Dynamics in Wuhan, China, of Novel CoronavirusInfected Pneumonia. The New England journal of medicine. 2020. https://doi.org/ 10.1056/NEJMoa2001316. Published online Jan 29, 2020.

10. Xie X, Zhong Z, Zhao W, Zheng C, Wang F, Liu J. Chest CT for Typical 2019-nCoV Pneumonia: Relationship to Negative RT-PCR Testing. Radiology. 2020. https://doi.org/ 10.1148/radiol.2020200343. Published online Feb 12, 2020.

11. Huang P, Liu T, Huang L, et al. Use of Chest CT in Combination with Negative RT-PCR Assay for the 2019 Novel Coronavirus but High Clinical Suspicion. Radiology. 2020.

https://doi.org/10.1148/radiol.2020200330. Published online Feb 12, 2020.

12. Chung M, Bernheim A, Mei X, et al. CT Imaging Features of 2019 Novel Coronavirus (2019-nCoV). Radiology. 2020. https://doi.org/10.1148/radiol.2020200230. Published online Feb 4, 2020.

13. Reittner P, Ward S, Heyneman L, Johkoh T, Müller NL. Pneumonia: high-resolution CT findings in 114 patients. Eur Radiol. 2003;13(3):515-21.

14. Cui J, Li F, Shi ZL. Origin and evolution of pathogenic coronaviruses. 2019;17(3):181-92.

15. Lee N, Hui D, Wu A, et al. A major outbreak of severe acute respiratory syndrome in Hong Kong. The New England journal of medicine. 2003;348(20):1986-94.

16. Wong KT, Antonio GE, Hui DSC, et al. Thin-section CT of severe acute respiratory syndrome: evaluation of 73 patients exposed to or with the disease. Radiology. 2003;228(2):395-400.

17. Antonio GE, Wong KT, Hui DSC, et al. Thin-section CT in patients with severe acute respiratory syndrome following hospital discharge: preliminary experience. Radiology. 2003;228(3):810-5. 
18. Kim EA, Lee KS, Primack SL, et al. Viral pneumonias in adults: radiologic and pathologic findings. Radiographics. 2002;22 Spec No:S137-S49.

19. Koo HJ, Lim S, Choe J, Choi S-H, Sung H, Do K-H. Radiographic and CT Features of Viral Pneumonia. Radiographics. 2018;38(3):719-39.

20. Ksiazek TG, Erdman D, Goldsmith CS, et al. A novel coronavirus associated with severe acute respiratory syndrome. The New England journal of medicine. 2003;348(20):1953-66.

21. Ooi GC, Khong PL, Müller NL, et al. Severe acute respiratory syndrome: temporal lung changes at thin-section CT in 30 patients. Radiology. 2004;230(3):836-44.

22. Grinblat L, Shulman H, Glickman A, Matukas L, Paul N. Severe acute respiratory syndrome: radiographic review of 40 probable cases in Toronto, Canada. Radiology. 2003;228(3):802-9.

\section{Tables}

Table 1 Clinical data and CT finding in initial symptom duration $<3$ days and $\geq 3$ days groups

\begin{tabular}{lccc}
\hline & $\begin{array}{c}\text { initial symptom } \\
\text { duration }<3 \text { days }\end{array}$ & $\begin{array}{c}\text { initial symptom } \\
\text { duration } \geq 3 \text { days }\end{array}$ & $\begin{array}{c}\text { P } \\
\text { value }\end{array}$ \\
\hline $\begin{array}{l}\text { gender } \\
\text { men }\end{array}$ & 10 & 10 & 0.722 \\
\hline$\quad$ women & 5 & 8 & 0.650 \\
\hline age(y) & $44.27 \pm 9.86$ & $45.83 \pm 9.42$ & 0.284 \\
\hline infection exposure history & 4 & 9 & 0.083 \\
$\quad$ No & 11 & 9 & 0.009 \\
$\quad$ Yes & $37.69 \pm 0.37$ & $38.01 \pm 0.72$ & \\
fever temperature $\left({ }^{\circ} \mathrm{C}\right)$ & $3.71 \pm 3.69$ & $7.21 \pm 4.96$ & \\
CT scores & $20.35 \pm 17.27$ & $31.10 \pm 15.96$ & 0.018 \\
length of the maximum & & & \\
inflammatory lesion $(\mathrm{mm})$ & & & \\
the number of affected & 10 & 9 & \\
pulmonary lobes & 5 & & \\
$\quad \leq 3$ & & &
\end{tabular}


Table 2 Spearman correlation analysis between the duration of initial symptoms and CT manifestations of lung lesions

\begin{tabular}{lll}
\hline & r value & P value \\
\hline Duration of initial symptoms and CT score & 0.502 & 0.003 \\
Duration of initial symptom and lesion size & 0.506 & 0.003 \\
\hline
\end{tabular}

\section{Abbreviations}

SARS-COV-2= Severe Acute Respiratory Syndrome Coronavirus 2

COVID-19= Corona Virus Disease 2019

2019-nCOV=2019 novel coronavirus

Figures 
54 patients were confirmed with the positive for SARS-COV-2 nucleic acid kit in the first and second affiliated hospital of Wenzhou medical university

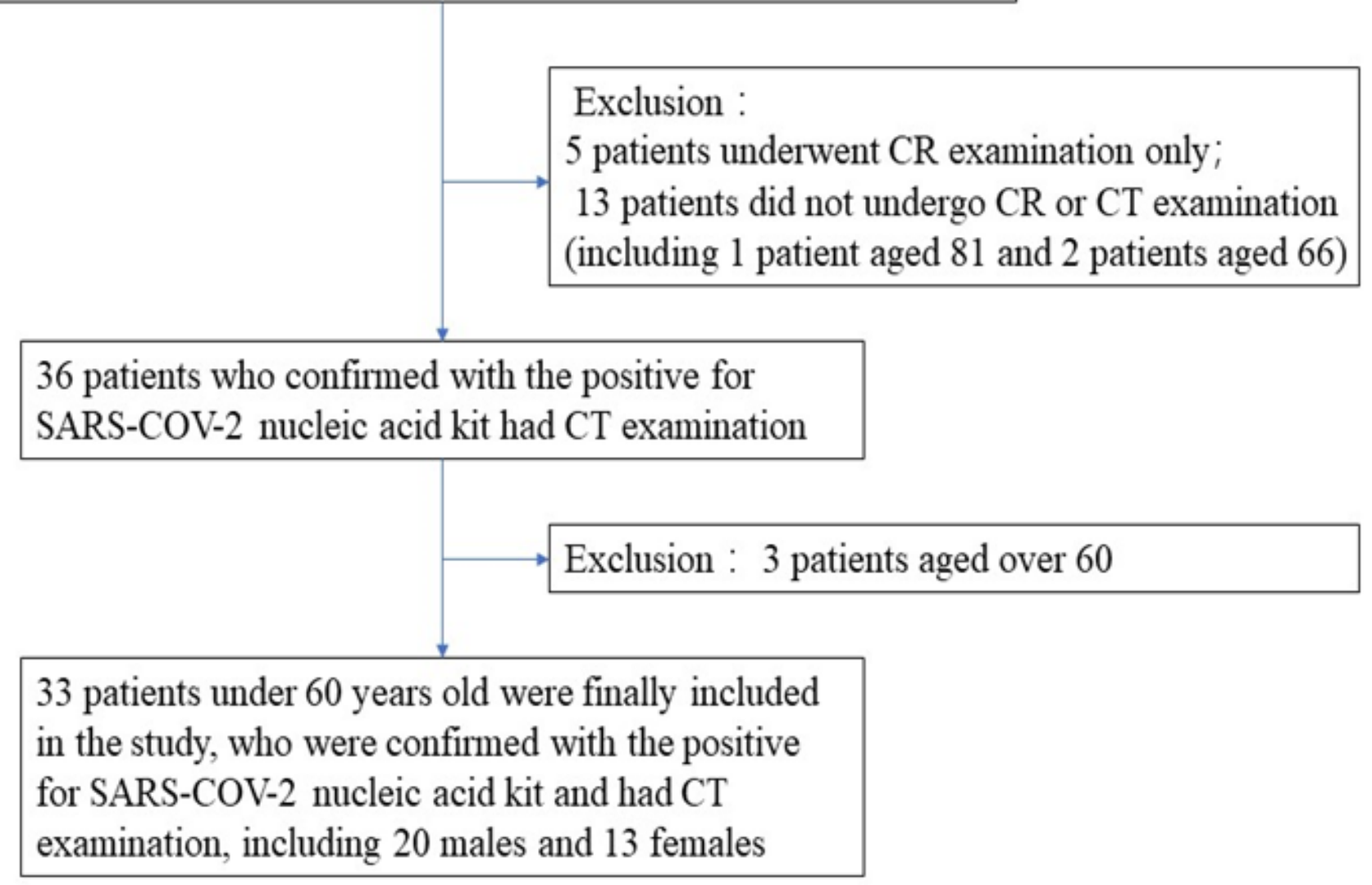

Figure 1

Flowchart of study population 


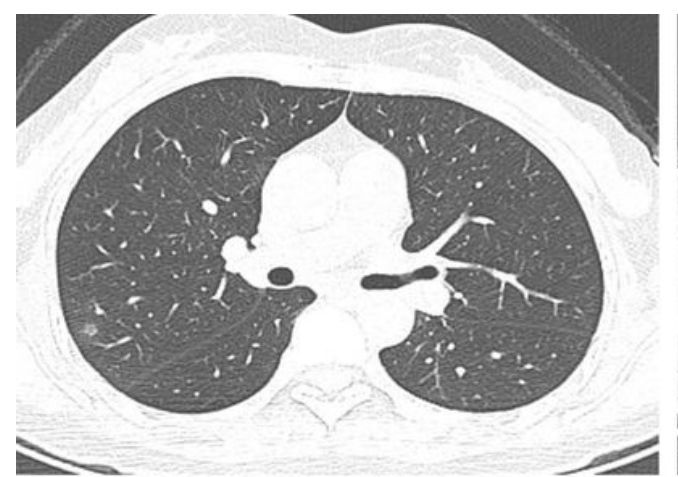

$A$

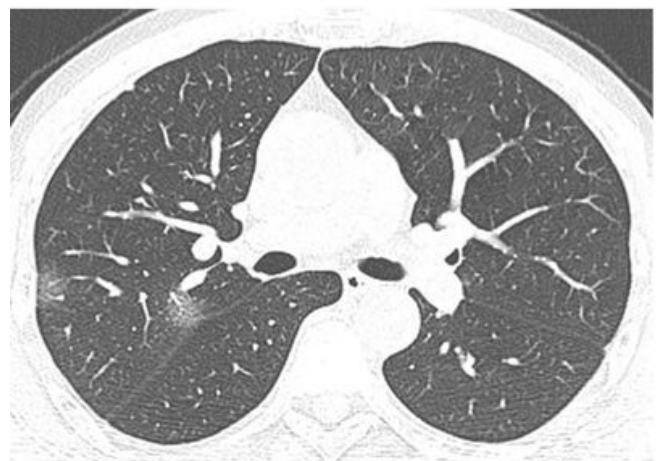

$D$

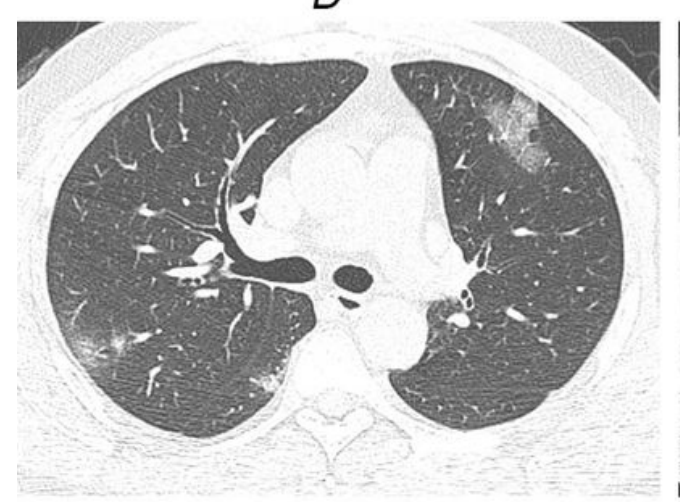

G

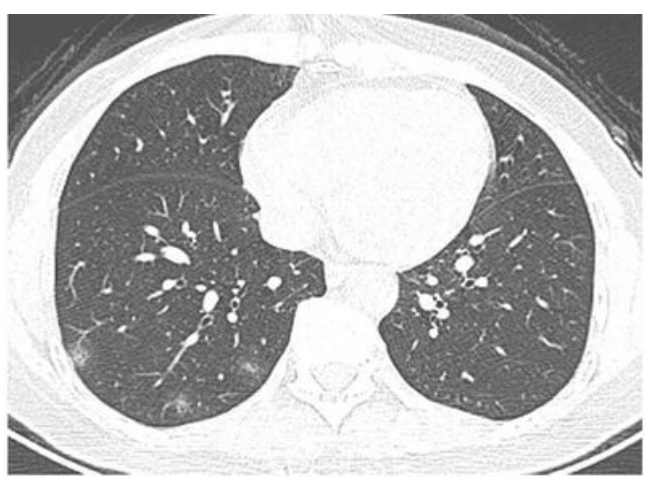

$B$

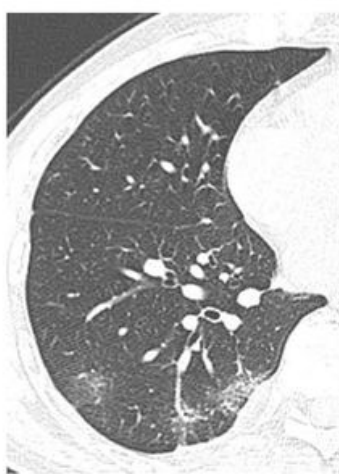

E

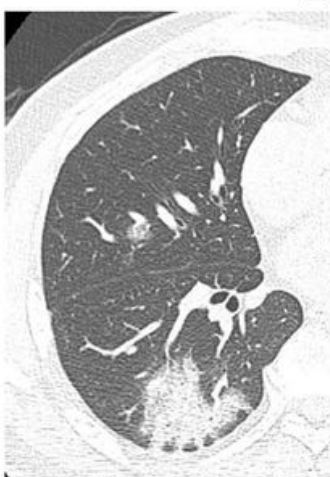

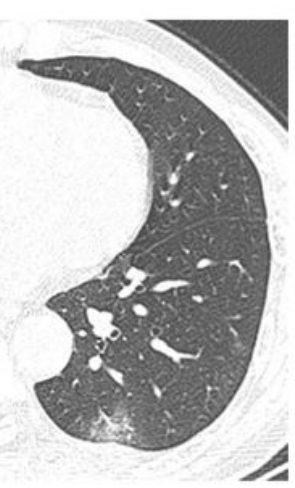

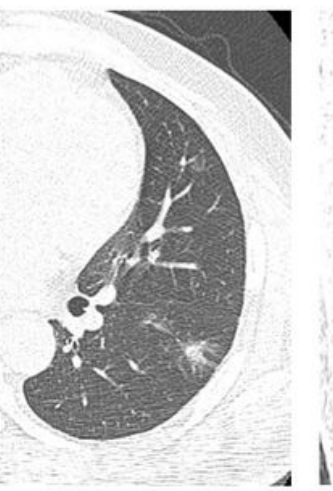

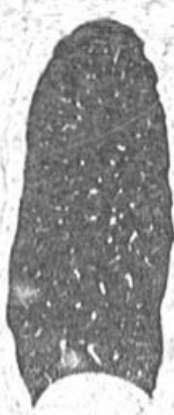

C

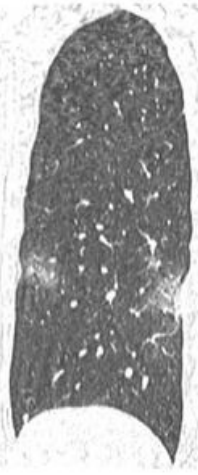

$F$

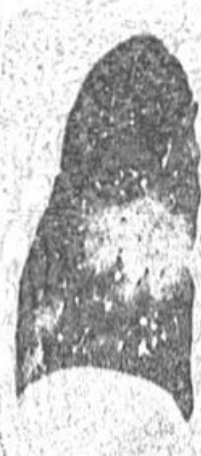

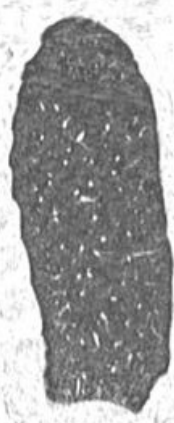

4
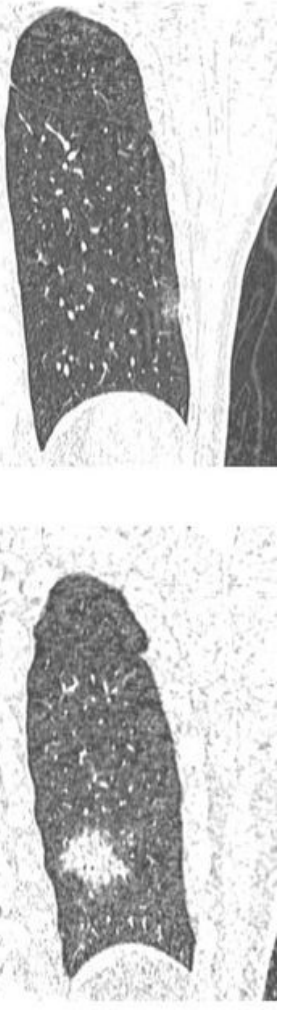

$H$

I

\section{Figure 2}

Chest plain CT imaging of the patients. (A-C) Case 1, male, 50 years old, has a history of infection exposure, fever for one day, CT finding was multiple round ground glass opacity, and CT score was 5. (DF) Case 2, male, 50 years old, has no history of infection exposure, fever for 2 days, CT finding showed multiple subpleural ground glass opacity, and CT score was 6 points. (G-I) Case 3, male, 46 years old, has a history of infection exposure, sore throat for 7 days, fever for 2 days, CT finding showed multiple patchy opacity in both lungs, the lesion in right lower lobe was mainly dominated consolidation, and CT score was 13 points. 